\title{
Adolescent Oral Methylphenidate Treatment Causes Post-administration Increase in Weight Gain and Hyperphagia with no Effect on Anxiety-related Behaviours in Rats
}

\author{
Omamuyovwi M. Ijomone ${ }^{1,2, *}$, Ugochukwu G. Esomonu ${ }^{1}$, Stephen O. Okori ${ }^{1}$, Christopher I. Eneji ${ }^{1}$ \\ ${ }^{1}$ Department of Anatomy, Faculty of Basic Medical Sciences, Cross River University of Technology, Nigeria \\ ${ }^{2}$ Department of Anatomical Sciences, School of Health and Health Technology, Federal University of Technology Akure, Nigeria
}

Copyright $(2016$ by authors, all rights reserved. Authors agree that this article remains permanently open access under the terms of the Creative Commons Attribution License 4.0 International License

\begin{abstract}
Methylphenidate (MPH) is a widely abused drug that has been shown to be a neural stimulant, amidst concern that it could be neurotoxic. The present study evaluated the changes in body weight, food intake and anxiety-related behaviours following oral administration of MPH to adolescent rats. Thirty- two (32) male adolescent wistar rats were divided into two groups and orally administered either saline vehicle or MPH from post-natal day (PD) 30 twice daily for 14 days. Body weight and food intake were monitored during administration and post-administration periods till PD 70. Anxiety-related activities were assessed using open-field (OF) and elevated plus maze (EPM) task on PD 45 and 70. Animals that received oral MPH administration showed significant decrease in weight gain but average food intake was not altered significantly during administration period. On the other hand, following discontinuation of $\mathrm{MPH}$ administration, treated rats recorded a significant increase in weight gain and average food intake. There was no significant treatment effect on central locomotion and time spent in open arms, which are indices of anxiety in the OF and EPM respectively. The study suggests that cessation of oral MPH treatments may induce a post-administration hyperphagia and a resultant effect of increased rate of weight gain. Also, the findings did not show anxiety modulatory actions of MPH following oral administration.
\end{abstract}

Keywords Methylphenidate, Adolescence, Body Weight, Open-field, Elevated-plus Maze

\section{Introduction}

Methylphenidate (MPH - Ritalin) is a neural stimulant prescribed for the treatment of attention - deficit/hyperactivity disorder (ADHD) $[1,2]$. ADHD is a constellation of symptoms including inattentiveness, impulsivity and hyperactivity that affects up to 18 percent of children ages 3-17 in the US [3, 4]. MPH acts as a dopamine transporter (DAT) blocker, which increases the level of dopaminergic neurotransmission in the brain [5]. DAT inhibition prevents reuptake of dopamine into presynaptic neurons at dopaminergic synapses. MPH also stimulates dopamine release into synapses, while also increasing the magnitude of dopamine release after a stimulus $[6,7]$.

There are concerns that frequent usage of MPH could be neurotoxic due to its tendency for abuse and addiction [8]. These concerns are even more pressing considering that MPH prescription is on the rise [1], hence researchers are continually investigating the long-term consequences of juvenile MPH treatment on brain and behaviour [9]. The Drug Enforcement Agency (DEA) have collected reports, some of which indicates that adolescents who are prescribed MPH may sell or give it to their classmates to ingest while others indicate "ADHD scams" in which a parent or any adult may present a child under their care to a number of doctors to obtain MPH prescription with claims that such child has ADHD [10].

The present study evaluated the effects of oral administration of MPH to adolescent rats on changes in body weight and food intake during adolescent stage and at adulthood. The study also assessed anxiety-related behaviours following such oral MPH treatments.

\section{Materials and Methods}

\subsection{Animal}

Thirty-two (32) Male albino wistar rats weaned at postnatal day (PD) 21 were housed in clean plastic cages of 4 
animals per cage and used for this study. Food and water was provided ad libitum. The animals were kept in clean environment at ambient room temperature. All experiments were conducted during the day (09:00-16:00 h) and all the experimental protocols were in accordance with National Institute of Health Guide for the Care and Use of Laboratory Animals (National Research Council, 2011), and approved by local Research Committee.

\subsection{Drug Administration}

On PD30 (this corresponds to beginning of adolescent stage in rats), a total of 32 rats were equally grouped into control and MPH-treated ( $\mathrm{n}=16$ rats each). The animals received oral administration of either saline vehicle only or MPH (Ritalin ${ }^{\circledR}$, Novartis Pharmaceuticals, Barbera del Valles, Spain; $5 \mathrm{mg} / \mathrm{kg}$ body weight dissolved in saline) twice daily for 14 days. Saline only treated animals served as control. Oral treatments were 4 hours apart with first administration at 09:00 h. At the start of administration on PD 30, average body weight of control group was $28.75 \pm 1.11$, and MPH group was $26.06 \pm 1.13$ (difference not statistically significant). On PD 45, some rats from each group $(\mathrm{n}=8)$ were subjected to behavioural studies. The remaining rats $(\mathrm{n}=8)$ were left till adulthood (PD70) and also subjected to behavioural studies. During the period of administration and post-administration, body weight of rats was monitored regularly every 3 days. Also body weight of rats was measured before behavioural studies on PD45 and 70. Weight gain during administration was obtained by subtracting initial body weight on PD30 from body weight on PD45. Weight gain during post-administration was obtained by subtracting body weight on PD45 from body weight on PD70. Daily food intake for each group was measured during and after administration.

\subsection{Behavioural Tests}

The behavioural studies carried out were the open-field (OF) and elevated-plus maze (EPM) tests. During the tests, the apparatus were cleaned with $10 \%$ ethanol between each rat. Behavioural studies were recorded using a digital camcorder and later scored manually and independently by at least two trained observers.

\subsubsection{Open-field}

The apparatus used has been previously described [15]. Rats were placed singly in the centre of the $\mathrm{OF}$, and the number of central squares (farther from walls) crossed, and peripheral squares (nearer to walls) that were crossed are noted for $5 \mathrm{~min}$.

\subsubsection{Elevated-plus Maze}

Apparatus used was as previously described [16]. Each rat was placed in the centre of the EPM facing an open arm, and allowed 5 minutes to freely explore. The parameters noted include; the number of entries and time spent in each type of arm. Arm entry is considered as when all four paws are within an arm.

\subsection{Statistical Analysis}

Data obtained for weight gain and food intake were analysed using student t-test. Data obtained from regular monitoring of body weight was analysed using two-way repeated measures ANOVA (treatment and time), with time as repeated measures. Data from behavioural studies were analysed using a two-way ANOVA for treatment and time factor. Bonferroni post-hoc was performed when appropriate. Statistical significance was taken set at $\mathrm{P}<0.05$ for all tests. Data are presented as means and SEM.

\section{Results}

\subsection{Body Weight and Food Intake}

The results of changes in body weight, weight gain and food intake are illustrated in Figure 1. For changes in body weight during administration period, two-way repeated measures ANOVA revealed significant treatment $[\mathrm{F}=11.08$; $\mathrm{P}<0.01]$, time $[\mathrm{F}=392.3 ; \mathrm{P}<0.001]$ and interaction $[\mathrm{F}=17.13$; $\mathrm{P}<0.001]$ effects. However, during post-administration period, two-way repeated measures ANOVA revealed significant time $[\mathrm{F}=474.7 ; \quad \mathrm{P}<0.001]$ and interaction $[\mathrm{F}=20.87 ; \mathrm{P}<0.001]$ effects, whereas treatment $[\mathrm{F}=2.08$; $\mathrm{P}=0.17]$ effect did not reach statistical significance. These data indicated that MPH administration lowered body weight during administration. However, on cessation of MPH administration, treated rats showed a rise in body weight towards values recorded for controls. Consequently, while t-test analysis reveals that body weight gain was significantly reduced $(\mathrm{P}<0.001)$ in $\mathrm{MPH}$ group during administration, body weight gain post-administration was significantly increased $(\mathrm{P}<0.001)$ in MPH group. In addition, difference in average food intake did not reach statistical significance during administration, however average food intake was significantly increased $(\mathrm{P}<0.001)$ post-administration. 

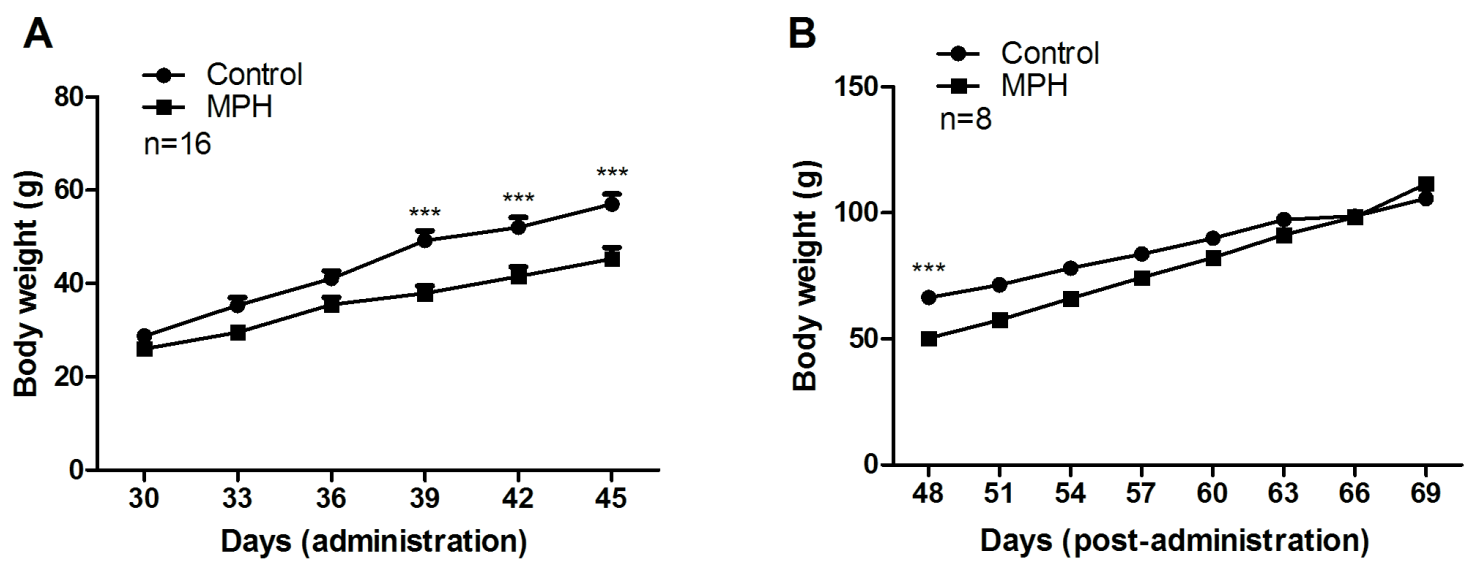

C

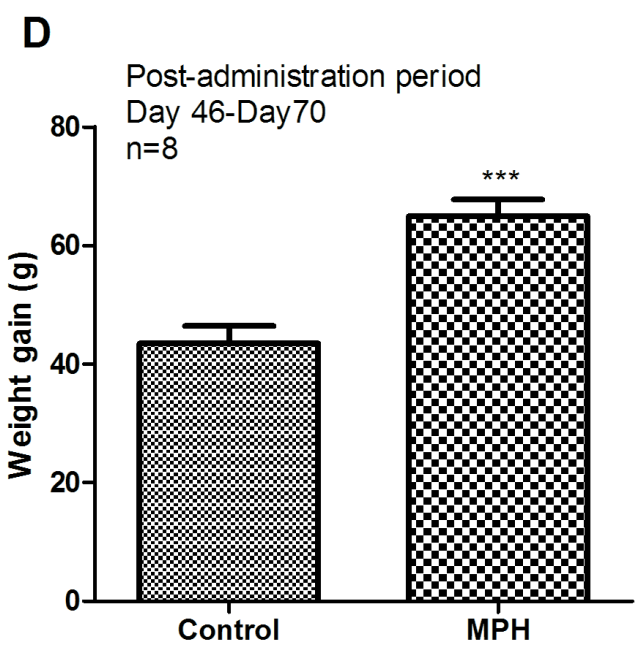

\section{E}

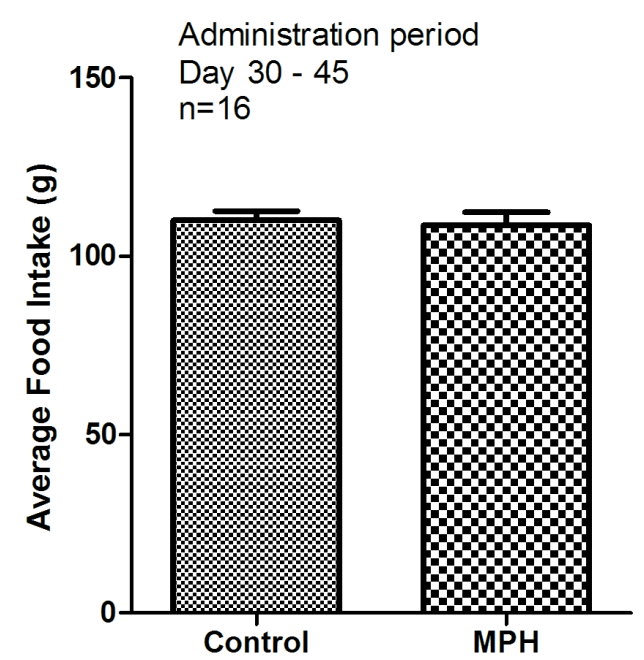

F

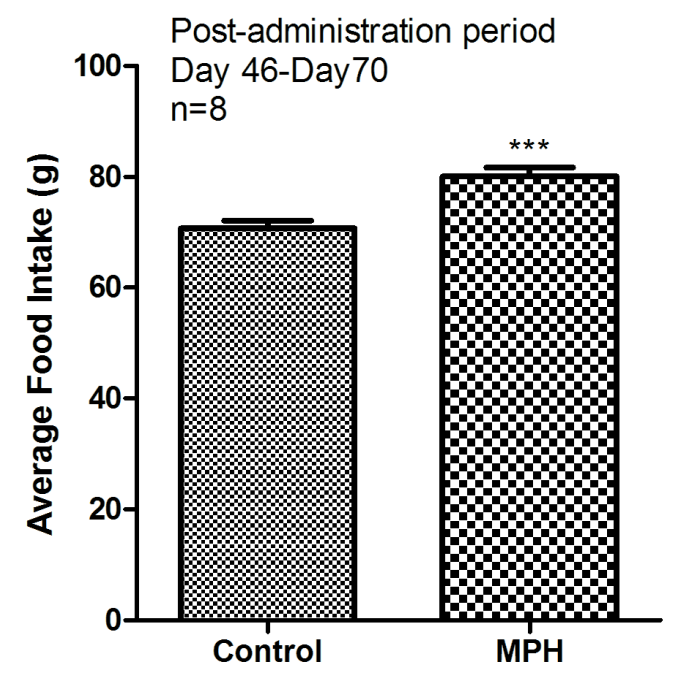

Figure 1. Effects of oral MPH administration on changes in body weight (A and B), weight gain (C and D) and average food intake (E and F). Two-way repeated measures ANOVA followed by Bonferroni post-tests for changes in body weight. Weight gain and Food intake analysed using student t-test. $* * * \mathrm{P}<0.001$ 


\subsection{Open-field}

The results for central and peripheral locomotion in the $\mathrm{OF}$ are illustrated in Figure 2. For central locomotion, two-way ANOVA revealed that differences between MPH and control did not reach statistical significance for treatment $[\mathrm{F}=2.36 ; \mathrm{P}=0.13]$, time $[\mathrm{F}=0.14 ; \mathrm{P}=0.71]$ and interaction $[\mathrm{F}=0.07 ; \mathrm{P}=0.79]$ effects. Also, for peripheral locomotion, two-way ANOVA revealed no significant difference for treatment $[\mathrm{F}=0.67 ; \mathrm{P}=0.42]$, time $[\mathrm{F}=1.05$; $\mathrm{P}=0.31]$ and interaction $[\mathrm{F}=0.56 ; \mathrm{P}=0.46]$ effects. These data indicated that oral MPH administration caused no significant changes in parameters scored in OF.

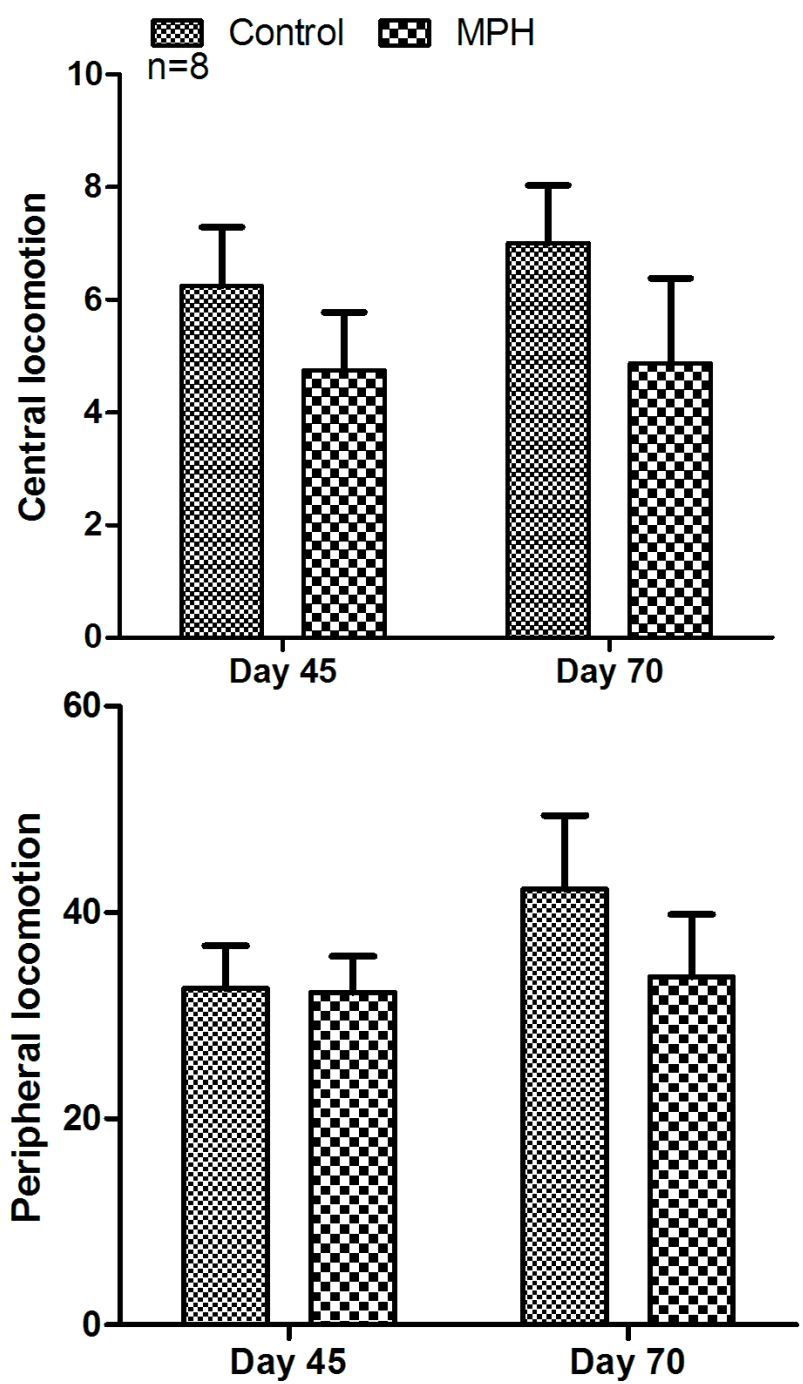

Figure 2. Central and peripheral locomotion in the OF task following oral MPH administration to adolescent rats. Two-way ANOVA for treatment and time factor followed by Bonferroni post-hoc.

\subsection{Elevated-plus Maze}

Figure 3 illustrates results for time spent in open arms and number of closed arm entries in the EPM. For time spent in open arms, two-way ANOVA showed significant time $[\mathrm{F}=34.66 ; \mathrm{P}<0.001]$ effect, however treatment $[\mathrm{F}=0.05$;
$\mathrm{P}=0.82]$ and interaction $[\mathrm{F}=0.002 ; \mathrm{P}=0.96]$ effects did not reach statistical significance. On the other hand, two-way ANOVA analysis of number of closed arm entries revealed significant treatment $[\mathrm{F}=18.38 ; \mathrm{P}<0.001]$ and interaction $[\mathrm{F}=4.60 ; \mathrm{P}<0.05]$ effects, whereas time $[\mathrm{F}=3.52 ; \mathrm{P}=0.07]$ effects did not reach statistical significance. Post hoc analysis of closed arm entries revealed a significant decrease $(\mathrm{P}<0.001)$ in MPH treated group compared to control on PD70. These data indicated while duration in open arms increased with time in both control and MPH groups, there was no difference between the two groups. Contrastingly, number of closed arm entries was lowered at PD70 by MPH treatment compared to control, but no time effect was observed.
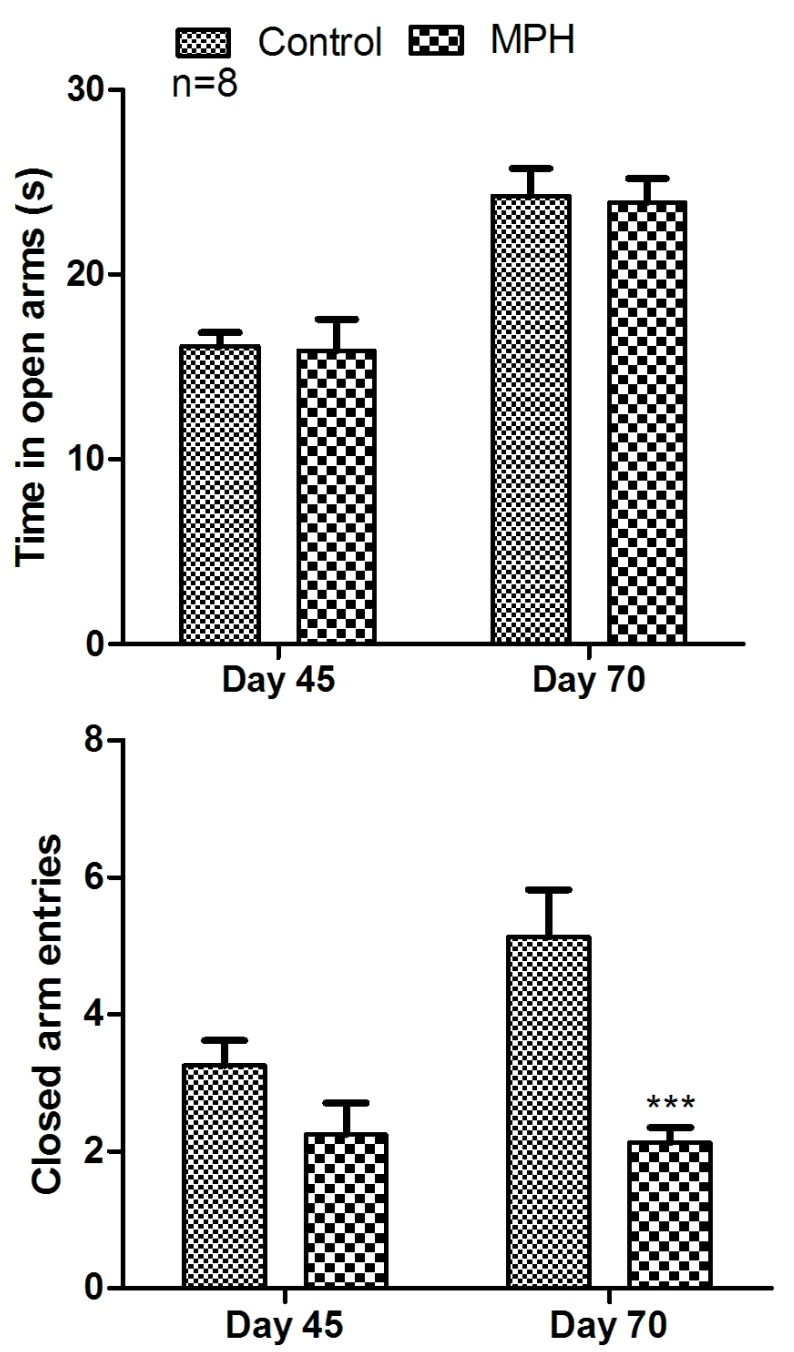

Figure 3. Time spent in open arms and number of closed arm entries in the EPM task following oral MPH administration to adolescent rats. Two-way ANOVA for treatment and time factor followed by Bonferroni post-hoc. $* * * \mathrm{P}<0.001$

\section{Discussion}

The psychostimulant MPH which has proven highly 
efficacious in preventing symptoms of ADHD is most commonly used in treatments of humans with this disorder. There are scientific concerns as to possible side-effects of MPH treatments on the adult brain and behaviour, especially following administrations during childhood and adolescence [1]. However, it is still unclear if juvenile MPH treatment directly affects behaviour seen in adults with ADHD. This study investigated the effects of oral administration of MPH to adolescent male rats on changes in body weight, food intake and anxiety-related activities during its administration and post-administration.

The present study reports that oral MPH decreases body weight of adolescent rats during its administration. However, on cessation of MPH treatment, there is a steady rise in body weight till adulthood. Consequently, while there is a decreased weight gain in adolescents following MPH treatment at end of administration, treated rats showed increased weight gain at adulthood. Strikingly, MPH did not alter food intake during administration, whereas MPH treatments stimulated food intake following its cessation. Previously, Gray et al., [11] showed that intraperitoneal injections of $5 \mathrm{mg} / \mathrm{kg}$ MPH to rats significantly slowed weight gain during pre-weaned period and early stages of adolescents. However, following discontinuation of MPH treatment at PD 35, these authors did not observe further significant difference in the rate and magnitude of weight gain [11]. In another study, intraperitoneally administered $\mathrm{MPH}$ at relatively high dose of $20 \mathrm{mg} / \mathrm{kg}$ to adult rats revealed significant decrease in body weight and average food intake [12]. Previous studies have reported the ability of MPH to sustain weight loss, mostly via inhibition of food intake $[13,14]$. Nevertheless, the present study suggests that decreased weight gain during MPH administration could be independent of food intake. Elfers and Roth [12] observed that weight loss due to MPH treatment is probably due to more than just decrease in food intake; suggesting that locomotor activity and period of sleep cycles may be puzzling dynamics in MPH induced weight loss due to its psychostimulant effects. Furthermore, these authors established that MPH could be used for the treatments of rat models of severe hypothalamic obesity (HO) by inhibiting sequelae presented in $\mathrm{HO}$ which includes rapid weight gain and hyperphagia [12]. While the present data supports reduced weight gain especially during oral administration of $\mathrm{MPH}$, discontinuation of such treatment may produce an opposing scenario as the present shows that MPH produces a withdrawal-phase induced hyperphagia and attendant increase in rate of weight gain in rats as they attain adulthood.

The present study shows that oral MPH administration did not alter parameters scored in the OF compared to control. Though the OF is mostly used to assess locomotor and exploratory activities [15], it was initially established as a test of emotionality [16]. In this sense, emotionality is scored based on tendency of the rodents to avoid the center of a large new area, while focusing their activities in the peripheral area. Therefore, central locomotion in the $\mathrm{OF}$ is basically seen as an index of experimental anxiety, whereas locomotion on the periphery is thought to denote general locomotion [17]. Considering the foregoing, the present data suggests that oral administration of MPH to adolescent rats does not alter anxiety-related behaviours in the OF during adolescent stage as well as at adulthood. This findings are consistent with Gray et al., [11], who also showed lack of anxiety related effects of MPH on rats in the OF task. Contrastingly, Vendruscolo et al., [16] reported MPH treated rats made fewer crossings in the central area. These authors suggested that MPH treatment during adolescence prompted anxious-like behaviours in the OF in adult rats. Furthermore, the present data did not reveal any treatment effect on time spent in open arms on the EPM. The EPM is used to more specifically assess anxiety-like behaviours in rats and duration in the open arms is the classic index of anxiety in this task [18]. Hence, the present study suggests that oral MPH administration did not also alter anxiety-related behaviours in the EPM during adolescent and in adulthood. In contrast, the earlier mentioned study of Gray et al., [11] reports that MPH treatment produced significant increase in time spent in open arms, while the earlier mentioned study by Vendruscolo et al., [16] showed no changes in this index of anxiety following MPH treatment. On the other hand, the present data revealed significant treatment effect on number of closed arm entries, which was reduced in MPH treatment group at Day 70. This parameter is associated with overall locomotor activity, as rats have a greater tendency to traverse the closed arms [19]. This suggests reduced locomotor activity at adulthood (on Day 70), in MPH treated rats; though this effect is not supported by changes in peripheral locomotion in the OF task which is more specifically an index of locomotor activity. Notwithstanding, if there is a decrease in overall locomotion when MPH is discontinued, such an effect may contribute to the observed increase in post-administration weight gain in the present study.

Comparing our data with the earlier mentioned studies, we observed that while Gray et al., [11] report anxiolytic effects of MPH on the EPM, Vendruscolo et al., [16] shows an anxiogenic effects on the OF. However, the present study did not observe any such anxiety modulating effects of MPH. It is noteworthy, that in these studies mentioned, MPH administration was via intraperitoneal injections whereas MPH was given orally in the present study. Thus oral MPH administration may have less effect on modulation of behaviours in rats. This is supported by the fact that intraperitoneal administrations are considered twice as potent as oral administration, especially in terms of altering neurotransmitter activities and stimulating behavioural changes [20]. Hence, in addition to the suggestion that MPH modulation of anxiety may be dependent on the task performed [16]; the present data also suggest that the route of administration may also affect the anxiety modulating effects of MPH.

In conclusion, the present findings indicate that while oral MPH may reduce rate of weight gain during administration period in adolescent rats, cessation of such treatments may 
induced a post-administration hyperphagia resulting in increased rate of weight gain. In addition, the findings suggest that administration of MPH orally do not produce anxiety modulatory effects.

\section{REFERENCES}

[1] Lagace DC, Yee JK, Bolaños CA, Eisch AJ. Juvenile administration of methylphenidate attenuates adult hippocampal neurogenesis. Biological Psychiatry 2006; 60:1121-1130.

[2] Hechtman L. Long-term treatment of children and adolescents with attention-deficit/hyperactivity disorder (ADHD). Curr Psychiatry Rep. 2006; 8:398-408.

[3] Dow-Edwards DL, Weedon JC, Hellmann E. Methylphenidate improves performance on the radial arm maze in periadolescent rats. Neurotoxicol \& Teratol. 2008; 30:419-427.

[4] Wilens TE, Dodson W. A clinical perspective of attention-deficit/hyperactivity disorder into adulthood. J Clin Psychiatry, 2004; 65:1301-1313.

[5] Volkow ND, Wang GJ, Fowler JS, Gatley SJ, Logan J, Ding YS, Hitzemann R, Pappas N. Dopamine transporter occupancies in the human brain induced by therapeutic doses of oral methylphenidate. Am J Psychiatry 1998; 155:1325-1331.

[6] Viggiano D, Vallone D, Sadile A. Dysfunctions in dopamine systems and ADHD: evidence from animals and modeling. Neural plasticity 2004; 11(1-2):97-114

[7] Gainetdinov RR, Caron MG. Genetics of childhood disorders: XXIV. ADHD, part 8: hyperdopaminergic mice as an animal model of ADHD. J Am Acad Child Adolesc Psychiatry 2001; 40:380-382.

[8] Zhu J, Reith M. Role of the dopamine transporter in the action of psychostimulants, nicotine, and other drugs of abuse. CNS; Neurol Dis-Drug Targets (Formerly Curr Drug Targets), 2008; 7:393-409.

[9] Andersen SL. Stimulants and the developing brain. Trends Pharmacol Sci. 2005; 26:237-243.

[10] Terrance W. DEA Congressional Testimony before the Committee on Education and the Workforce: Subcommittee on Early Childhood, Youth, and Families. 2000. [http://www.dea.gov/pubs/cngrtest/ct051600.htm]

[11] Gray JD, Punsoni M, Tabori NE, Melton JT, Fanslow V, Ward MJ, Zupan B, Menzer D, Rice J, Drake CT. Methylphenidate administration to juvenile rats alters brain areas involved in cognition, motivated behaviors, appetite, and stress. J Neurosci. 2007; 27:7196- 7207.

[12] Elfers CT and Roth CL. Effects of methylphenidate on weight gain and food intake in hypothalamic obesity. Frontiers in Endocrinol. 2011, 2:Article 78

[13] LeddyJJ, Epstein LH, Jaroni JL, Roemmic JN, Paluch RA, Gold- field GS, Lerman C. Influence of methylphenidate on eating in obese men. Obes. Res. 2004; 12:224-232.

[14] Leddy JJ, Waxmonsky JG, Salis RJ, Paluch RA, Gnagy EM, Mahaney P, Erbe R, Pelham WE. Epstein LH. Dopamine-related genotypes and the dose-response effect of methylphenidate on eating in attention-deficit/hyperactivity disorder youths. J. Child Adolesc. Psychopharmacol. 2009; 19:127-136.

[15] Ijomone OM, Olaibi OK, Biose IJ, Mba C, Umoren KE, Nwoha PU. Performance on motor associated behavioural tests following chronic nicotine administration. Annals Neurosci. 2014; 21(2):42-46.

[16] Vendruscolo LF, Izıdio GS, Takahashia RN, Ramos A. Chronic methylphenidate treatment during adolescence increases anxiety-related behaviors and ethanol drinking in adult spontaneously hypertensive rats. Behav Pharmacol. 2008; 19:21-27.

[17] Prut L, Belzung C. The open field as a paradigm to measure the effects of drugs on anxiety-like behaviors: a review. Eur J Pharmacol. 2003; 463:3-33.

[18] Ijomone OM, Olaibi OK, Mba C, Biose IJ, Tete SA, Nwoha PU. Chronic nicotine administration does not alter cognitive or mood associated behavioural parameters. Pathophysio. 2015; 22(1):57-63.

[19] Lopez LLH, Caif F, Garca S, Fraile M, Landa AI, Baiardi G, Lafuente JV, Braszko JJ, Bregonzio C, Gargiulo PA. Anxiolytic-like effect of losartan injected into amygdala of the acutely stressed rats. Pharmacol Rep. 2012; 64: 54-63.

[20] Gerasimov MR, Franceschi M, Volkow ND, Gifford A, Gatley SJ, Marsteller D, Molina PE, Dewey SL. Comparison between intraperitoneal and oral methylphenidate administration: a microdialysis and locomotor activity study. J Pharmacol Exp Ther. 2000; 295:51-57. 